\title{
Ovarian Hyperandrogenism
}

National Cancer Institute

\section{Source}

National Cancer Institute. Ovarian Hyperandrogenism. NCI Thesaurus. Code C120146.

Increased production of androgens by the ovaries. 